Grant/research support from: Inserm, the Arthritis Foundation, the French Society of Rheumatology, Pfizer and the Région Pays de la Loire (Bioregate)

\section{P061 TARGETING B-CELL ACTIVATING FACTOR (BAFF) IMPAIRS ECTOPIC LYMPHONEOGENESIS IN MURINE MODELS OF SJÖGREN'S SYNDROME}

IJ Campos*, ${ }^{2} \mathrm{~T}$ Slocombe, ${ }^{1} \mathrm{~S}$ Nayar, ${ }^{1} \mathrm{~V}$ lannizzotto, ${ }^{1} \mathrm{DH}$ Gardner, ${ }^{1} \mathrm{CD}$ Buckley, ${ }^{2} \mathrm{~A}$ Haynes, ${ }^{2} \mathrm{RB}$ Henderson, ${ }^{1} \mathrm{~F}$ Barone. ${ }^{1}$ Institute of Inflammation and Ageing, University of Birmingham, Birmingham; ${ }^{2}$ Immunoinflammation TAU, GSK, Stevenage, UK

\subsection{6/annrheumdis-2018-EWRR2018.80}

Introduction Tertiary lymphoid structures (TLS) characterised by germinal centre formation and B cell proliferation represent the histological hallmark of primary Sjögren's syndrome (pSS). However, the events preceding the formation of such ectopic structures and factors driving their persistence are unknown. Overexpression of BAFF, also known as B cell lymphocyte stimulator (BLyS), in pSS patients has been linked with the presence of autoreactive B cells and autoantibody production. ${ }^{1}$ Furthermore, in pSS salivary glands BAFF is associated with the expansion of specific B cell subsets, and with B cell repopulation post rituximab treatment. ${ }^{2}$

Objectives In this work we aimed to dissect the dynamics of B cell subsets within tertiary lymphoid structures following BAFF-targeted treatment in both inducible and chronic animal models that mimic the histological features of pSS.

Methods Submandibular salivary glands of C57BL/6 mice were intra-ductally cannulated with luciferase-encoding replicationdeficient adenovirus to induce TLS formation as previously described. $^{3}$ Prior to salivary gland cannulation, mice were treated with two doses (i.p.) of either anti-BLyS mAb or isotype control. Salivary glands were dissected at day 15 postcannulation and TLS formation in both groups was assessed. NOD.B10.H2b mice were similarly treated with anti-BLyS $\mathrm{mAb}$ at 26 weeks old and salivary gland infiltrates assessed 21 days later.

Results Histological analysis of salivary glands from anti-BLyS treated C57BL/6 animals unveiled severely compromised TLS formation. Post anti-BLyS treatment, salivary glands were infiltrated by $\mathrm{T}$ cell clusters but only few, and scattered, B cells were present, contrasting with fully developed and organised TLS in the salivary glands of mice treated with isotype control. Significantly lower numbers of B cells, particularly from the B2 subset, as well as plasmablasts, infiltrated salivary glands of anti-BLyS treated mice. However, treatment with anti-BLyS did not affect numbers of infiltrating $\mathrm{T}$ cells (both CD4 and CD8), proliferative $\mathrm{T}$ cells, or plasma cells in inflamed salivary glands. In a chronic setting, salivary glands from NOD.B10.H2b mice were also infiltrated by significantly lower numbers of B2 B cells following anti-BLyS treatment.

Conclusions Our data highlights BAFF as a key player in ectopic lymphoneogenesis during inflammation as well as a subset-specific role for BAFF in B cell maturation. Furthermore, these results support future studies of BAFF-targeted therapeutics in pSS.

\section{REFERENCES}

1. Pers, et al. Ann N Y Acad Sci 2005.

2. Pers, et al. Arthritis Rheum 2007.

3. Bombardieri, Barone, et al. Jl 2012.
Acknowledgements This research is funded by GSK.

Disclosure of interest J. Campos: None declared, T. Slocombe Employee of: GSK, S. Nayar: None declared, V. Iannizzotto: None declared, D. Gardner: None declared, C. Buckley: None declared, A. Haynes Employee of: GSK, R. Henderson Employee of: GSK, F. Barone: None declared

\section{P062 SIGNIFICANT DECREASE OF T-CELLS BUT NOT MACROPHAGES IN THE SYNOVIUM OF PATIENTS WITH ACTIVE RHEUMATOID ARTHRITIS AFTER TREATMENT WITH TOCILIZUMAB}

K Chatzidionysiou*, M Engström, E af Klint, A Hensvold, Al Catrina. Rheumatology Department, Karolinska Institute, Stockholm, Sweden

\subsection{6/annrheumdis-2018-EWRR2018.81}

Introduction Tocilizumab (TCZ) is an anti-IL6R monoclonal antibody approved for the treatment of Rheumatoid Arthritis (RA). There is limited data on synovial tissue histology changes.

Objectives The aim of this study was to evaluate the effect of TCZ on synovial cell populations and on citrullination.

Methods 15 patients with definite RA, according to ACR 1987 criteria, independent of disease duration, were included. Synovial biopsies were obtained before and after 8 weeks of treatment with TCZ from all patients. We evaluated by immunohistochemistry (IHC) expression of citrullinated proteins (CP) and protein arginine deiminase (PAD) enzymes in synovial tissue before and after treatment with TCZ (1325:C03, 1325:B09, PAD2, PAD4). Negative controls were used for each antibody. Expression of CD68, CD3, CD20 and CD55 was also evaluated. Evaluation of all IHC variables was performed by two blinded independent observers using a semiquantitative score on a $0-3$ scale (0, no staining; 1 , low amounts of staining; 2, moderate amounts of staining; 3 , high amounts of staining). Paired-wised Wilcoxon Signed Ranks Test was used to compare the median value before and after treatment.

\begin{tabular}{llll}
\multicolumn{4}{c}{ Abstract P062 Table 1 } \\
\hline & Basline & 12 weeks & Wilcoxonsigned ranks test \\
\hline DAS28 BL & $5.9(4.7-6.8)$ & $2.98(2.0-3.8)$ & 0.028 \\
SJC BL & $9(3-14)$ & $1(0-4.25)$ & 0.009 \\
TJC BL & $10(4-15)$ & $1(0-2.25)$ & 0.017 \\
ESR BL & $34(15-69)$ & $6(5-16)$ & 0.001 \\
CRP BL & $11(5-27)$ & $1(1-2)$ & 0.005 \\
CD68 & $2(1-3)$ & $2(0.75-3)$ & 0.41 \\
CD3 & $2(1-3)$ & $1(0-2)$ & 0.046 \\
CD55 & $2(2-3)$ & $1.5(1-2)$ & 0.05 \\
C03 (cit) & $1(0-1)$ & $0(0-1)$ & 0.025 \\
B09 (cit) & $2(1-2)$ & $2(1-2)$ & 0.20 \\
PAD2 & $3(2-3)$ & $2(1.5-3)$ & 0.16 \\
PAD4 & $2(2-3)$ & $2(2-3)$ & 0.37 \\
\hline
\end{tabular}

Results The median (IQR) age, disease duration, N. prior biologic DMARDs and DAS28 at baseline was 66 (58-79), 4 (113), 1 (0-2), 6 (5-7), respectively. 93\% were female, 53\% were RF + and 60\% ACPA+, 53\% had concomitant glucocorticoids and only $27 \%$ had concomitant conventional synthetic DMARDs. Significant reductions in DAS28, swollen and 